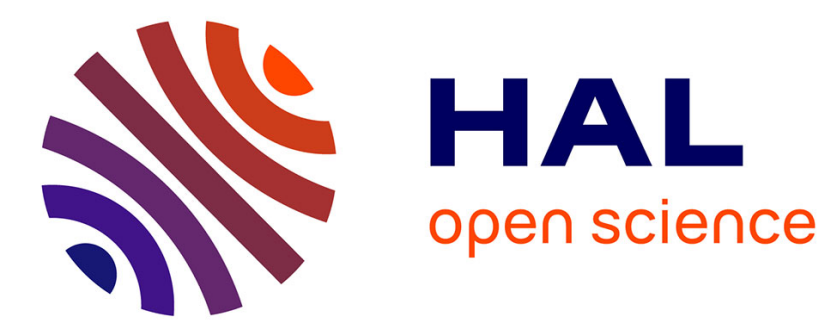

\title{
Analyse du pouvoir thermoélectrique dans Pb1-xSn xTe
} M. Ocio

\section{To cite this version:}

M. Ocio. Analyse du pouvoir thermoélectrique dans Pb1-xSn xTe. Revue de Physique Appliquée, 1972, 7 (3), pp.151-154. 10.1051/rphysap:0197200703015100 . jpa-00243612

\section{HAL Id: jpa-00243612 https://hal.science/jpa-00243612}

Submitted on 1 Jan 1972

HAL is a multi-disciplinary open access archive for the deposit and dissemination of scientific research documents, whether they are published or not. The documents may come from teaching and research institutions in France or abroad, or from public or private research centers.
L'archive ouverte pluridisciplinaire HAL, est destinée au dépôt et à la diffusion de documents scientifiques de niveau recherche, publiés ou non, émanant des établissements d'enseignement et de recherche français ou étrangers, des laboratoires publics ou privés. 


\title{
REVUE DE PHYSIQUE APPLIQUÉE
}

\author{
Supplément au «Journal de Physique »
}

Classification

Physics Abstracts

$17.26-17.40$

\section{ANALYSE DU POUVOIR THERMOÉLECTRIQUE DANS Pb $_{1-x} \mathrm{Sn}_{x} \mathrm{Te}$}

\author{
M. OCIO \\ Service d'Electronique Physique, Centre d'Etudes Nucléaires de Saclay \\ 91-Gif-sur-Yvette, France
}

(Reçu le 14 janvier 1972)

\begin{abstract}
Résumé. - L'analyse de Rogers du pouvoir thermoélectrique sur la base d'un modèle à double bande de valence est étendue pour inclure l'anisotropie de la bande non parabolique. La nouvelle formulation est appliquée à l'analyse des résultats obtenus sur deux alliages $\mathrm{Pb}_{1-x} \mathrm{Sn}_{x} \mathrm{Te}$. Des valeurs sont estimées pour certains paramètres de bande, en particulier pour l'écart d'énergie entre les deux bandes de valence.

Abstract. - Rogers' analysis of the Seebeck coefficient on the basis of a two valence band model is extended by introducing the anisotropy parameter of the non parabolic band. Experimental results on two $\mathrm{Pb}_{1-x} \mathrm{Sn}_{x}$ Te alloys are analysed using the new formulation. Values of band parameters, specially the energy gap between the two valence bands are estimated.
\end{abstract}

Les propriétés électriques d'alliages $\mathrm{Pb}_{1-x} \mathrm{Sn}_{x} \mathrm{Te}$ de type $p$ ont permis de souligner l'existence dans ces matériaux de deux bandes de valence [1]-[4]. Rogers a développé un modèle à double bande de valence [5], [6], la première bande non parabolique étant conforme au modèle de Cohen [7] dans le cas de la symétrie de rotation et de réflexion. Ce modèle a été appliqué à l'étude de la variation du pouvoir thermoélectrique en fonction de la concentration apparente en porteurs à $300^{\circ} \mathrm{K}$ dans $\mathrm{SnTe}$ [5], d'une part et dans deux alliages $\mathrm{Pb}_{1-x} \mathrm{Sn}_{x} \mathrm{Te}$ [8] d'autre part.

Cependant, dans sa formulation, Rogers utilise un certain nombre d'approximations dans le but de pallier l'absence de données relatives à l'anisotropie de la première bande de valence dans SnTe.

On dispose à l'heure actuelle de résultats concernant cette anisotropie dans $\mathrm{Pb}_{1-x} \mathrm{Sn}_{x}$ Te. Nous avons donc étendu l'analyse de Rogers en incluant le paramètre d'anisotropie $K_{n}=m_{\mathrm{ln}} / m_{\mathrm{ln}}$ où $m_{\mathrm{n}}^{\prime}$ est la masse effective longitudinale en bord de bande, et $m_{\text {tn }}$ la masse effective transversale en bord de bande. La nouvelle formulation a été appliquée aux résultats obtenus sur $\mathrm{Pb}_{1-x} \mathrm{Sn}_{x} \mathrm{Te}$.

Dans un semiconducteur comportant deux bandes de valence, la concentration apparente en porteurs $p^{*}$ et le pouvoir thermoélectrique $\alpha$ sont donnés par les relations usuelles :

$$
p^{*}=\frac{p_{1}(1+t b)^{2}}{r_{1}\left(1+s t b^{2}\right)},
$$

REVUE DE PHYSIQUE APPLIQUÉE. - T. 7, No 3, SEPTEMBRE 1972 et

$$
\alpha=\frac{1+t b \alpha_{2}}{1+t b}
$$

avec

$$
s=\frac{r_{2}}{r_{1}}, \quad t=\frac{p_{2}}{p_{1}} \quad \text { et } \quad b=\frac{\mu_{2}}{\mu_{1}} .
$$

$r_{i}, p_{i}, \mu_{i}, \alpha_{i}$, sont respectivement le facteur d'anisotropie de Hall, la concentration en porteurs, la mobilité et le pouvoir thermoélectrique de la $i$-ième bande.

$\mathrm{La}$ première $\mathrm{b}$ bande de valence $\mathrm{BV}_{1}$ est supposée suivre la loi de dispersion donnée par Cohen dans le cas de la symétrie de rotation et de réflexion :

$$
E=\frac{\hbar^{2} k_{\mathrm{t}}^{2}}{2 m_{\mathrm{tn}}}\left[\frac{1}{1+\frac{E}{E_{\mathrm{G}}}+\frac{\hbar^{2} k_{\mathrm{l}}^{2}}{2 m_{\mathrm{ln}}^{\prime} E_{\mathrm{G}}}}\right]+\frac{\hbar^{2} k_{1}^{2}}{2 m_{\mathrm{ln}}},
$$

où $k_{\mathrm{t}}$ et $k_{1}$ sont respectivement les nombres d'onde transverse et longitudinal, $m_{\mathrm{t}}$ et $m_{1}$ les masses effectives transverse et longitudinale de la bande de valence $\mathrm{BV}_{1}$, et $m_{1}$, la masse effective de la bande de conduction BC. L'indice $\mathrm{n}$ indique qu'il s'agit des masses en bord de bande. $E_{\mathrm{G}}$ est la largeur de la bande interdite. Dans le cas d'une forte dégénérescence, la concentration en porteurs a pour expression :

$$
p_{1}=\frac{8}{3 \sqrt{\pi}}\left(\frac{2 \pi m_{\mathrm{dn}_{1}} k T}{h^{2}}\right)^{3 / 2} \eta^{3 / 2}\left[1+A\left(E_{\mathrm{F}}\right)\right],
$$


où $\eta=E_{\mathrm{F}} / k T$ est le niveau de Fermi réduit

avec

$$
A\left(E_{\mathrm{F}}\right)=\left(1+\frac{u}{5}\right) \frac{\eta}{\eta_{\mathrm{G}}}
$$

$$
\eta_{\mathrm{G}}=\frac{E_{\mathrm{G}}}{k T} \quad \text { et } \quad u=\frac{m_{\mathrm{ln}}}{m_{\mathrm{l}^{\prime} \mathrm{n}}}
$$

et $m_{\mathrm{dn}_{1}}=N_{\mathrm{V}_{1}}^{2 / 3}\left(m_{\mathrm{tn}_{1}}^{2} \cdot m_{\mathrm{ln}_{1}}\right)^{1 / 3}, N_{\mathrm{V}_{1}}$ étant le nombre de vallées équivalentes.

Dans le cas de la diffusion des porteurs par les phonons acoustiques, Rogers calcule la mobilité à partir du temps de relaxation donné par Ziman [9] :

$$
\tau(E)=\frac{h d V^{2}}{2 \pi^{2} \varepsilon k T \mathcal{N}(E)},
$$

où $d$ est la densité, $V$ la vitesse du son, $\varepsilon$ le potentiel de déformation et $\mathcal{N}(E)$ la densité d'états dans une seule vallée. La mobilité est tirée de la relation classique :

$$
\mu=\frac{e \tau}{m_{\mathrm{i}}},
$$

où $m_{\mathbf{i}}$ est la masse d'inertie :

$$
\frac{3}{m_{\mathrm{i}}}=\frac{2}{m_{\mathrm{t}}}+\frac{1}{m_{1}}
$$

Nous introduisons ici le facteur d'anisotropie ${ }^{[} K_{n}$ : la masse d'inertie s'écrit alors :

$$
m_{\mathrm{i}}=\frac{3 K_{n} m_{\mathrm{t}}}{2 K_{n}+1+5 / 3 A\left(E_{\mathrm{F}}\right)},
$$

et l'expression de la mobilité devient :

$$
\begin{aligned}
\mu_{1}=Q N_{\mathrm{V}_{1}}^{2} T^{-1} m_{\mathrm{dn}_{1}}^{-3} & m_{\mathrm{ln}_{1}}^{1 / 2} \times \\
& \times E_{\mathrm{F}}^{-1 / 2} \frac{2 K_{n}+1+5 / 3 A\left(E_{\mathrm{F}}\right)}{3 K_{n}\left[1+5 / 3 A\left(E_{\mathrm{F}}\right)\right]^{2}},
\end{aligned}
$$$$
\text { où } Q \text { est égal à }
$$

$$
\frac{e h^{4} d V^{2}}{2^{9 / 2} \pi^{3} \varepsilon_{k}^{2}}
$$

Le calcul du pouvoir thermoélectrique est effectué en utilisant la relation donnée par Kolodziejczack et Zukotynski [10] pour un matériau fortement dégénéré :

$$
\alpha= \pm \frac{k \pi^{2}}{3 e} \cdot k T \cdot\left(\frac{1}{\mu} \cdot \frac{\mathrm{d} \mu}{\mathrm{d} E}+\frac{3}{2} \cdot \frac{1}{\gamma} \cdot \frac{\mathrm{d} \gamma}{\mathrm{d} E}\right)_{E_{\mathrm{F}}}
$$

$\mu$ est la mobilité et $\gamma$ la loi de dispersion non parabolique exprimée sous la forme :

$$
a_{\beta \delta} k_{\beta} k_{\delta}=\gamma(E) \text {. }
$$

Bien que la loi de dispersion de Cohen ne puisse se réduire à une expression de cette forme, on peut effectuer le calcul en utilisant la relation, [6] :

$$
\left(\frac{3}{2} \cdot \frac{1}{\gamma} \cdot \frac{\mathrm{d} \gamma}{\mathrm{d} E}\right)_{E_{\mathrm{F}}}=\left(\frac{g^{(E)}}{p}\right)_{E_{\mathrm{F}}},
$$

où $g(E)$ est la densité d'état de la bande.
Après calcul de $\mathrm{d} \mu / \mathrm{d} E$, on obtient :

$$
\alpha_{1}= \pm \frac{k \pi^{2}}{3 e} \cdot \frac{1}{\eta} \times
$$

$$
\times\left(\frac{1+2 A\left(E_{\mathrm{F}}\right)}{1+A\left(E_{\mathrm{F}}\right)}-\frac{(X-1) \cdot\left(4 K_{n}+X\right)}{X\left(2 K_{n}+X\right)}\right),
$$

où $X=1+5 / 3 A\left(E_{\mathrm{F}}\right)$.

La deuxième bande de valence $\mathrm{BV}_{2}$ est supposée parabolique; elle comporte $N_{\mathrm{V}_{2}}$ vallées de masse effective $m_{2}^{*}$. L'écart d'énergie entre $B V_{1}$ et $B V_{2}$ est $E_{\mathrm{V}}$. La concentration en porteurs $p_{2}$, la mobilité $\mu_{2}$ et le pouvoir thermoélectrique $\alpha_{2}$ sont donnés par les relations usuelles dans le cas d'une bande parabolique et d'une diffusion des porteurs par les phonons acoustiques :

$$
\begin{aligned}
& p_{2}=\frac{4}{\sqrt{\pi}}\left(\frac{2 \pi m_{\mathrm{d}_{2}}}{\hbar^{2}}\right)^{3 / 2} F_{1 / 2}\left(\eta-\eta_{\mathrm{V}}\right), \\
& \mu_{2}=Q N_{\mathrm{V}_{2}}^{2} T^{-1} m_{\mathrm{d}_{2}}^{-3} \cdot m^{* 1 / 2} E^{-1 / 2} \\
& \alpha_{2}=\frac{k}{e}\left[\frac{2 F_{1}\left(\eta-\eta_{\mathrm{V}}\right)}{F_{0}\left(\eta-\eta_{\mathrm{V}}\right)}-\left(\eta-\eta_{\mathrm{V}}\right)\right]
\end{aligned}
$$

avec :

$$
m_{\mathrm{d}_{2}}=N_{\mathrm{V}_{2}}^{2 / 3} m_{2}^{*} \quad \text { et } \quad \eta_{\mathrm{v}}=\frac{E_{\mathrm{V}}}{k T}
$$

$F_{i}(x)$ est l'intégrale de Fermi d'ordre $i$ et $E_{\mu}$ est une énergie moyenne pour les trous lourds.

On calcule enfin le rapport des mobilités $b=\mu_{2} / \mu_{1}$ en tenant compte des relations :

$$
\begin{aligned}
m_{\mathrm{ln}_{1}} & =\left(\frac{K_{n}}{N_{\mathrm{V}_{1}}}\right)^{2 / 3} m_{\mathrm{dn}_{1}}, \\
m_{2}^{*} & =\left(\frac{1}{N_{\mathrm{V}_{2}}}\right)^{2 / 3} m_{\mathrm{dn}_{2}} .
\end{aligned}
$$

On obtient :

$$
\begin{aligned}
b=\left(\frac{N_{\mathrm{V}_{2}}}{N_{\mathrm{V}_{1}}}\right)^{5 / 3}\left(\frac{m_{\mathrm{dn}_{1}}}{m_{\mathrm{dn}_{2}}}\right)^{5 / 2} & \left(\frac{\eta}{x}\right)^{1 / 2} \times \\
& \times\left[\frac{3 K_{n}^{2 / 3}\left(1+5 / 3 A\left(E_{\mathrm{F}}\right)^{2}\right)}{2 K_{n}+2+5 / 3 A\left(E_{\mathrm{F}}\right)}\right],
\end{aligned}
$$

où $x$ est un paramètre qui prend la valeur 1,5 pour $E_{\mathrm{F}}-E_{\mathrm{V}} \leqslant 3 / 2 k T$ et la valeur : $\left(E_{\mathrm{F}}-E_{\mathrm{V}}\right) / k T$ pour $E_{\mathrm{F}}-E_{\mathrm{V}}>3 / 2 k T$.

Pour ne pas faire intervenir l'anisotropie, Rogers effectue le calcul de $\mu_{1}$ en supposant $m_{\mathrm{t}} \ll m_{1}$ et calcule $b$ en posant $m_{\mathbf{l n}_{1}}=m_{2}^{*}$.

On peut noter que, dans le modèle de Cohen, alors que la masse effective longitudinale est constante, la masse effective transversale augmente avec l'énergie. Même si $m_{\mathrm{tn}_{1}} \ll m_{\mathrm{ln}_{1}}$, on ne peut plus poser $m_{\mathrm{t}} \ll m_{1}$, dès que $E_{\mathrm{F}}$ devient grand. Par exemple, si l'on prend pour, $u$ la valeur 1 , la valeur de $m_{\mathrm{t}}$ pour $E_{\mathrm{F}}=E_{\mathrm{g}}$ est triple de sa valeur pour $E_{\mathrm{F}}=0$. Rogers constate d'ailleurs que le meilleur accord avec les résultats 
expérimentaux est obtenu quand on modifie l'expression de $b$ de telle manière que $m_{1}=m_{\mathrm{t}}$. Or, les surfaces de Fermi de la première bande de valence de SnTe sont de forme allongée, même par des concentrations en porteurs supérieures à $10^{20} \mathrm{~cm}^{-3}$ [11].

D'autre part, en considérant $m_{\mathrm{In}_{1}}=m_{2}^{*}$, et compte tenu des valeurs choisies de $m_{\mathrm{dn}_{1}}$ et $m_{\mathrm{dn}_{2}}$, Rogers adopte implicitement une valeur 10 pour l'anisotropie en bord de $\mathrm{BV}_{1}$; ceci apparaît comme étant en désaccord avec la condition $m_{\mathrm{t}} \ll m_{1}$. Aussi avons-nous introduit le facteur d'anisotropie qui permet une plus grande cohérence dans la formulation.

Nous avons analysé sur la base du modèle étendu les résultats de mesures électriques effectuées sur des échantillons de $\mathrm{Pb}_{0,72} \mathrm{Sn}_{0,28} \mathrm{Te}$ et $\mathrm{Pb}_{0,53} \mathrm{Sn}_{0,47} \mathrm{Te}$ dopés au $\mathrm{Na}$, et dont les concentrations apparentes à $300^{\circ} \mathrm{K}$ sont comprises entre $10^{19}$ et $8 \times 10^{20} \mathrm{~cm}^{-3}$.

Dans un précédent travail [8], les valeurs des fractions molaires $x$ pour les deux solutions solides avaient été prises égales à 0,3 et 0,5 après mesure par diffraction de rayons $\mathrm{X}$. Les présentes valeurs résultent d'une analyse à la sonde électronique. De plus, des échantillons nouveaux de $\mathrm{Pb}_{0,72} \mathrm{Sn}_{0,28} \mathrm{Te}$ ont été élaborés afin de permettre une meilleure définition de la courbe expérimentale.

Récemment, Melngailis et coll. [12] ont évalué le coefficient d'anisotropie de $\mathbf{B V}_{1}$ dans les alliages $\mathrm{Pb}_{1-x} \mathrm{Sn}_{x} \mathrm{Te}$ riches en $\mathrm{PbTe}(x \leqslant 0,315)$. Compte tenu des concentrations en porteurs dans les échantillons de ces auteurs, on peut considérer que les valeurs publiées sont proches de celles du coefficient en bord de bande $K_{n}=m_{\mathrm{ln}} / m_{\mathrm{tn}}$. Pour tous ces échantillons, $m_{\mathrm{l}} / m_{\mathrm{t}}$ a une valeur voisine de 10 , et il ne semble pas que l'anisotropie varie avec $x$. D'autre part, notons que de faibles variations de $K_{n}$ autour d'une valeur moyenne n'ont pas une grande influence sur le résultat du calcul. Nous avons adopté pour $K_{n}$ la valeur 10 et pris pour certains autres paramètres les valeurs suivantes :

$N_{\mathrm{V}_{1}}=4[12] ; \quad N_{\mathrm{V}_{2}}=12[13] ; \quad r_{2}=1 ; u=1$.

Les valeurs de $E_{\mathrm{G}}$ sont tirées des résultats déjà connus [14], [15]. Nous avons choisi pour $m_{\mathrm{dn}_{1}}$ des valeurs légèrement inférieures à celles que le modèle parabolique permet de calculer [1]. Le meilleur accord des courbes calculées avec les résultats expérimentaux est obtenu pour les valeurs suivantes des paramètres.

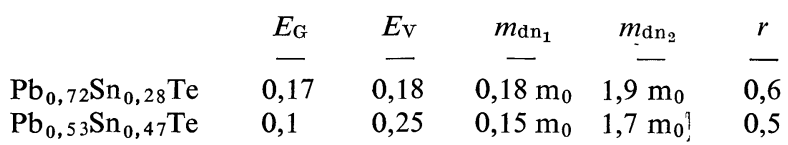

Les courbes calculées sont représentées figure 1 . Pour comparaison nous avons fait figurer les courbes calculées, pour les mêmes valeurs des paramètres, d'après le modèle initial de Rogers.

Les valeurs de $E_{\mathrm{G}}$ et $E_{\mathrm{V}}$ à $300^{\circ} \mathrm{K}$ pour les deux alliages étudiés, ainsi que les données similaires de la littérature pour d'autres solutions, sont indiquées figure 2. Les écarts d'énergie entre les bords de la bande de conduction et des bandes de valence sont donnés pour les alliages riches en $\mathrm{Pb}$, par rapport au bord de la bande $\mathrm{BV}_{1}$, lequel, suivant le modèle d'inversion des bandes [14] devient pour les alliages riches en $\mathrm{Sn}$, le bord de la bande de conduction. En prenant pour PbTe la valeur $E_{\mathrm{V}}=0,05 \mathrm{eV}$ et pour SnTe une valeur de $E_{\mathrm{v}}$ proche de $0,3 \mathrm{eV}$ (voir réf. [5]), ces résultats permettent d'envisager une variation approximativement linéaire avec $x$, de l'écart d'éner-

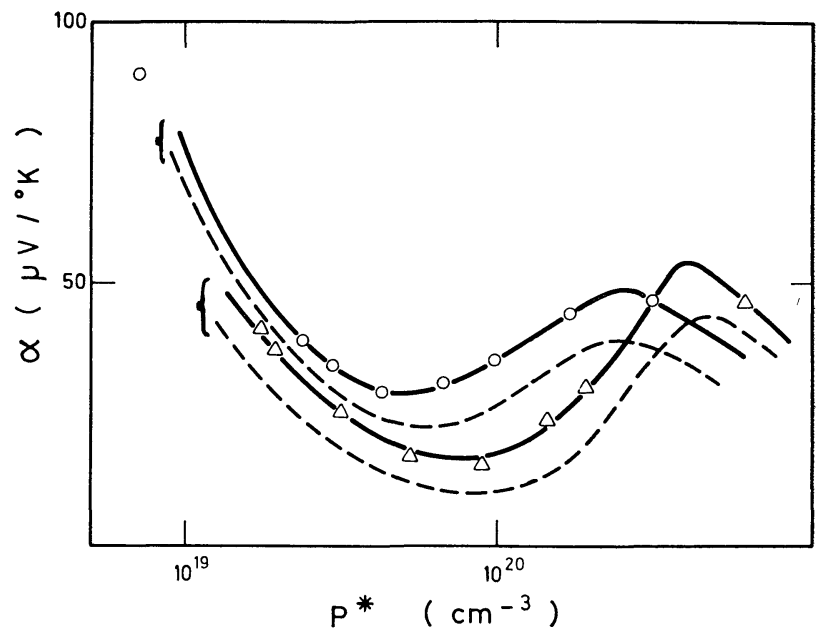

FIG. 1. - Variation du pouvoir thermoélectrique à $300^{\circ} \mathrm{K}$ en fonction de la concentration apparente en porteurs dans $\mathrm{Pb}_{0,72} \mathrm{Sn}_{0,28} \mathrm{Te}(0)$ et $\mathrm{Pb}_{0,53} \mathrm{Sn}_{0,47} \mathrm{Te}(\Delta)$. Les courbes correspondent aux valeurs calculées selon le présent modèle (et selon le modèle original de Rogers (- - - ) pour les mêmes valeurs des paramètres.

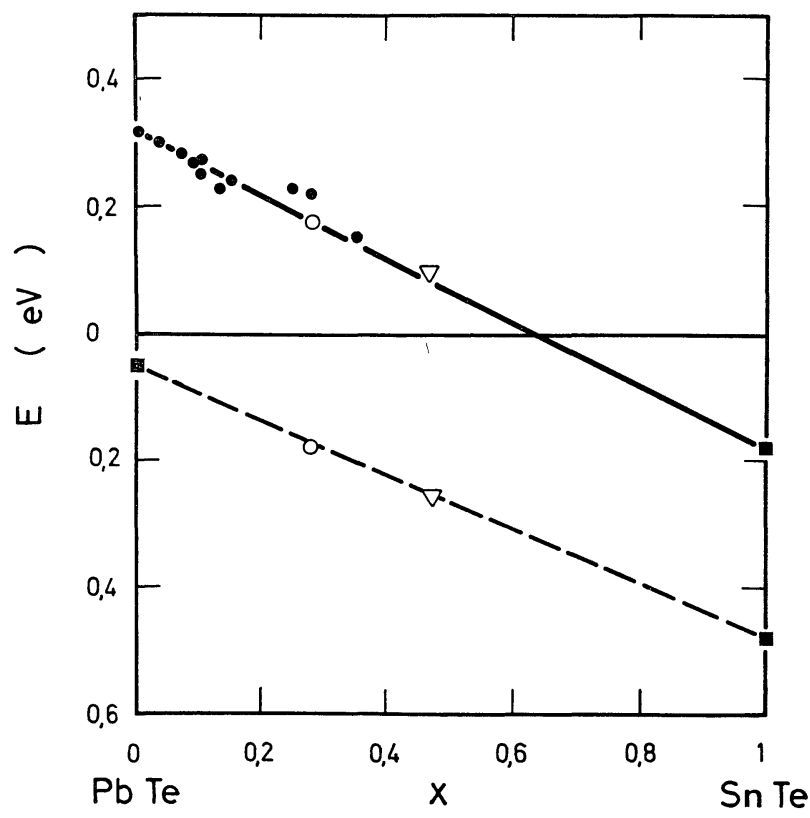

FIG. 2. - Variation à $300^{\circ} \mathrm{K}$ de la bande interdite $E_{\mathrm{G}}$ (——) et variation suggérée de l'écart $E_{\mathrm{V}}$ d'énergie entre les deux bandes de valence (-- - - ) dans $\mathrm{Pb}_{1-x} \mathrm{Sn}_{x} \mathrm{Te}$, en tonction de la fraction molaire $x(\bullet:$ voir références dans $[14] ; \square:$ voir références dans $[5] ; 0, \nabla:$ présent travail). 
gie entre $\mathrm{BV}_{2}$ et l'axe de référence pour les énergies. Ceci est corroboré par une étude récente de la structure de bande dans le système $\mathrm{Pb}_{1-x} \mathrm{Sn}_{x} \mathrm{Te}$ [16].
Remerciements. - Je remercie Messieurs H. J. Albany et R. S. Allgaier pour d'utiles discussions et correspondance.

\section{Bibliographie}

[1] Efimova (B. A.) et Kolomoetz (L. A.), Fiz. Tverd. Tela, 1965, 7, 424.

[2] Tauber (R. N.) et Cadoff (I. B.), J. Appl. Phys., 1967, 38, 3714.

[3] Ocio (M.) et Albany (H. J.), Phys. Letters, 1968, 27A, 72.

[4] Colloque Intern. sur les composés IV-VI, Gif-surYvette, 1968, J. Physique, 1968, 29, C 4.

[5] Rogers (L. M.), Brit. J. Appl. Phys., 1968, 1, 845.

[6] Rogers (L. M.), Brit. J. Appl. Phys., 1967, 8, 1227.

[7] Cohen (M. H.), Phys. Rev., 1961, 121, 387.

[8] Ocio (M.) et Albany (H. J.), Phys. Letters, 1969, 30A, 169.

[9] Ziman (J. M.), Electrons and Phonons (Oxford University Press, London, 1960).
[10] Koldziejczack (J.) et Zukotynski (S.), Phys. Stat. Solidi, 1964, 5, 145.

[11] Burke (J. R.), Jr., Houston (B.), Savage (H. T.), BABISKIN (J.), SiEBENMANN (P. G.), International Conference on the Physics of Semiconductors, Kyoto 1966, J. Phys. Soc. of Japan, 1966, 21, Suppl.

[12] Melngailis (J.), Harman (T. C.), Mavroides (J. G.), Dimmock (J. O.), Phys. Rev., 1971, B3, 370.

[13] Crocker (A. T.) et Rogers (L. M.), Colloque International sur les composés IV-VI, Gif-sur-Yvette 1968, J. Physique, 1968, 29, C4.

[14] Dimmock (J. O.), Melngailis (I.), Strauss (A. J.), Phys. Rev. Letters, 1966, 16, 1193.

[15] Dixon (J. R.) et Bis (R. F.), Phys. Rev., 1968, 176, 942.

[16] Allgaier (R. S.) et Houston (B.) Phys. Rev., 1972, 5, 2186. 